\title{
THE EFFECT OF SPONTANEOUS ELECTROMECHANICAL ACTIVITY ON THE METABOLISM OF ACETYLCHOLINESTERASE IN CULTURED EMBRYONIC RAT MYOTUBES $^{1}$
}

\author{
STEVEN K. BROCKMAN, LINDA H. YOUNKIN, AND STEVEN G. YOUNKIN² \\ Department of Pharmacology, Case Western Reserve University School of Medicine, Cleveland, Ohio 44106
}

Received April 28, 1983; Revised July 29, 1983; Accepted August 2, 1983

\begin{abstract}
We have investigated the effect of electromechanical activity on the molecular forms of acetylcholinesterase (AChE) in cultured embryonic rat myotubes. Both globular and asymmetric forms of AChE are present on the 5th day of culture when myotubes are just beginning to fibrillate. Between days 5 and 8 , the $4 \mathrm{~S}\left(\mathrm{G}_{1}\right), 10 \mathrm{~S}\left(\mathrm{G}_{4}\right)$, and $16 \mathrm{~S}\left(\mathrm{~A}_{12}\right)$ forms increase dramatically, and appreciable $12.5 \mathrm{~S}\left(\mathrm{~A}_{8}\right) \mathrm{AChE}$ appears. When fibrillation is prevented by adding tetrodotoxin on day 4, the increases in the $A_{12}$ and $A_{8}$ forms are prevented, and the increases in the $G_{4}$ and $G_{1}$ forms are significantly impaired. At 8 days, fibrillating myotubes have 19 times more $A_{12} \mathrm{AChE}$ and over 4 times more $G_{1}$ and $G_{4}$ enzyme than do nonfibrillating myotubes. The effect of tetrodotoxin is reversible. When tetrodotoxin is removed at 7 days, fibrillation resumes promptly, and globular and asymmetric forms recover. Light microscopic examination of fibrillating and nonfibrillating myotubes showed that tetrodotoxin does not affect the gross morphological development of the myotubes. Titration of AChE-active sites with $O$-ethyl- $S^{2}$-diisopropyl methyl-phosphonothionate demonstrated that the increase in AChE activity associated with fibrillation is due to an increase in the number of $\mathrm{AChE}$ molecules present and not to an increase in the rate at which individual AChE molecules turn over acetylcholine. To evaluate AChE metabolism in fibrillating and nonfibrillating myotubes, we examined the enzyme after inactivating it with paraoxon. Paraoxon readily penetrates cells and diethylphosphorylates a serine in the active site of AChE, thereby inactivating it. The diethylphosphorylated enzyme is stable, but it can be reactivated rapidly and quantitatively with pyridine-2-aldoxime methiodide (2-PAM). After inactivating AChE with paraoxon, we simultaneously evaluated synthesis (by following the newly synthesized active AChE) and turnover (by following the 2-PAM-reactivatable $\mathrm{AChE}$ ). Our results show that globular and asymmetric forms of AChE are both synthesized more rapidly in fibrillating than in nonfibrillating myotubes.
\end{abstract}

A central concern of cell biology is the regulation of cell surface macromolecules. One cell surface protein that is of considerable interest is the enzyme acetylcholinesterase (AChE). There are at least six molecular forms of this enzyme. Three of these forms are globular proteins and three are asymmetric (Bon et al., 1979). The globular forms of the enzyme are monomers, dimers, or tetramers of identical catalytic subunits designated $\mathrm{G}_{1}, \mathrm{G}_{2}$, and $\mathrm{G}_{4}$.

\footnotetext{
${ }^{1}$ We wish to thank Dr. T. L. Rosenberry for his advice throughout this study and for his comments on the manuscript. We also wish to thank Dr. J. Massoulie for the generous gift of MTP used in this study. This investigation was supported by National Institutes of Health Grant NS 15219, by National Institutes of Health Medical Scientist Training Grant GM-07250 (S. B.), and by a grant from the Muscular Dystrophy Association.

${ }^{2}$ To whom correspondence should be addressed.
}

The asymmetric forms are designed $\mathrm{A}_{4}, \mathrm{~A}_{8}$, and $\mathrm{A}_{12}$ and consist of one, two, or three tetramers attached to an elongated collagen-like tail. Muscle cells externalize three types of AChE: globular forms that are secreted, globular forms that are integral sarcolemmal proteins, and asymmetric forms that appear to associate electrostatically with the basal lamina that surrounds each muscle fiber (Rotundo and Fambrough, 1980; Younkin et al., 1982). In all cells that have been studied, a large percentage of total $\mathrm{AChE}$ is intracellular (Lazar and Vigny, 1980; Rotundo and Fambrough, 1980; Inestrosa et al., 1981; Taylor et al., 1981; Brockman et al., 1982). Recent studies by Rotundo and Fambrough (1980) on cultured embryonic chick myotubes indicate that this intracellular AChE is newly synthesized enzyme that is destined for externalization.

Most of the AChE in innervated adult mammalian 
skeletal muscle is intracellular $\mathrm{G}_{1}$ and sarcolemmal $\mathrm{G}_{4}$ enzyme that is located outside neuromuscular junctions (nonjunctional $\mathrm{AChE}$ ), but a significant fraction (26\% in rat diaphragm) is highly concentrated at neuromuscular junctions with its active sites exposed (Younkin et al., 1982). This junctional AChF is the functionally important enzyme in skeletal muscle that rapidly hydrolyzes the acetylcholine released from motoneuron terminals thereby terminating neuromuscular transmission. Asymmetric forms are of particular interest because they comprise virtually all ( $84 \%$ in adult rat diaphragm) of the functionally important enzyme (Younkin et al., 1982).

The interaction between the $\alpha$-motoneuron and the skeletal muscle fiber that it innervates is one of the most intensively studied examples of mammalian cell-cell interaction. $(x$-Motoneurons profoundly influence both junctional $\left(\mathrm{A}_{12}\right.$ and $\left.\mathrm{A}_{8}\right)$ and nonjunctional (intracellular $\mathrm{G}_{1}$ and sarcolemmal $\mathrm{G}_{4}$ ) AChE, and there is evidence suggesting that innervation also influences the enzyme that is secreted by muscle cells (Inestrosa ct al., 1977; Carter and Brimijoin, 1981). Thus this system is a good one for inquiring generally into the mechanisms that are involved in the regulation of cell surface proteins and more particularly into the mechanisms by which neurons regulate the functionally important surface proteins of the cells that they innervate.

The influence of the $\alpha$-motoneuron on skeletal muscle $\mathrm{AChE}$ must begin with one or several signals that pass between the $r$-motoneuron and the skeletal muscle fiber. These signals must (through what may be a very complicated series of cellular events) alter the metabolism of the enzyme in a way that generates the levels and cellular localization of the various forms of the enzyme found in adult innervated muscle. There is good evidence that the electromechanical activity (action potentials and/or contraction) set up in muscle by $\alpha$-motoneurons is one important signal that mediates the influence of $\alpha$-motoneurons on both junctional (Lomo and Slater, 1980; Rubin et al., 1980) and nonjunctional (Drachman, 1972) AChE in skeletal muscle fibers. The electromechanical activity set up in muscle by nerve also profoundly reduces the number of acetylcholine receptors in nonjunctional sarcolemma (Lomo and Rosenthal, 1972), and there is good evidence that it does so by reducing the rate at which acetylcholine receptors are synthesized (Reiness and Hall, 1977; Linden and Fambrough, 1979).

The aim of this study was to examine the influence of electromechanical activity on AChE metabolism, focusing particularly on the influence of activity on the rate at which the various forms of the enzyme are synthesized. We have carried out this study in cultured embryonic rat myotubes. These cells are not innervated and have no endplates, but they provide a very useful model system in which to study the effect of electromechanical activity on $\mathrm{AChE}$ metabolism. Myotubes derived from 18- to 20day rat embryos contain both globular and asymmetric forms of AChE (Koenig and Vigny, 1978). The myotubes undergo spontaneous electromechanical activity (fibrillation) that can be inhibited conveniently with tetrodotoxin (TTX), a drug that specifically inactivates the voltage-dependent sodium channels in the sarcolemma that are required for action potential generation.
The spontaneous electrical activity (fibrillation) occurring in these cells is clearly intense enough to influence AChE metabolism. Rieger et al. (1980) have shown that inhibition of fibrillation with TTX reversibly prevents the development of the $A_{12}$ form of the enzyme.

We have compared fibrillating rat myotubes with those in which fibrillation was blocked with TTX. Our results indicate that the levels of both globular and asymmetric forms of $\mathrm{AChE}$ are higher in fibrillating myotubes and that this occurs because of an increase in the rate at which the globular and asymmetric forms are synthesized. It appears, therefore, that the electromechanical activity set up in muscle by nerve can regulate the synthesis of functionally important surface proteins in either a positive (AChE) or negative (acetylcholine receptor) direction.

\section{Materials and Methods}

Myotube culture. Cultures were prepared from the leg musculature of 20-day rat fetuses. Pregnant female rats were anesthetized with ether and the fetuses were removed using aseptic technique. Skinned hindlimbs then were separated from each fetus at midthigh, and the feet were removed. Dissected legs were stored at $0^{\circ} \mathrm{C}$ in Hanks' balanced salt solution without calcium or magnesium (HBSS) until all dissection was complete. After dissection, each leg was rinsed in HBSS and cut into six to eight pieces with scissors. These pieces were pooled, 25 to $30 \mathrm{ml}$ of $0.05 \%$ collagenase in Puck's balanced salt solution $\left(0.137 \mathrm{M} \mathrm{NaCl}, 5.4 \mathrm{mM} \mathrm{KCl}, 1.1 \mathrm{mM} \mathrm{KH}_{2} \mathrm{PO}_{4}\right.$, $2.0 \mathrm{mM} \mathrm{Na} \mathrm{HPO}_{4}, 0.11 \mathrm{mM} \mathrm{CaCl}, 0.06 \mathrm{mM} \mathrm{MgSO}_{4} \mathrm{pH}$ 7.4) were added, and the preparation was incubated at $37^{\circ} \mathrm{C}$ for $30 \mathrm{~min}$. Prior to incubation and at 15 and 30 min, the tissue was aspirated mechanically by pipette for 1 to $2 \mathrm{~min}$ to facilitate dissociation. After dissociation, the cells were filtered through gauze, and the filtrate was supplemented with $20 \mathrm{ml}$ of culture medium (Dulbecco's modified Eagle's medium containing $10 \%$ fetal calf serum, 50 units $/ \mathrm{ml}$ of penicillin, and $50 \mu \mathrm{g} / \mathrm{ml}$ of streptomycin) and centrifuged at $500 \times g$ for $5 \mathrm{~min}$. The cells then were resuspended in culture medium at a density of 700,000 cells $/ \mathrm{ml}$, and $7.5-\mathrm{ml}$ aliquots were seeded into $100-\mathrm{mm}$ tissue culture dishes (Falcon) coated with $1 \%$ gelatin. Each fetus yielded about 1.5 cultures. Cultures were maintained at $37^{\circ} \mathrm{C}$ in an atmosphere of $10 \% \mathrm{CO}_{2}$ and $90 \%$ air. Medium was changed on day 4. After changing the medium, TTX (final concentration $1 \mu \mathrm{M}$ ) was added to some of the cultures to prevent the myotubes from fibrillating. In some experiments TTX was added on day 7 (with no medium change) to stop fibrillation of actively fibrillating myotubes. Cells were washed and harvested at the times indicated in each experiment. Washing consisted of rinsing three times with phosphate-buffered saline (PBS; $137 \mathrm{~mm} \mathrm{NaCl}, 1.1$ $\mathrm{mM} \mathrm{KH} \mathrm{PO}_{4}, 2.0 \mathrm{mM} \mathrm{Na}_{2} \mathrm{HPO}_{4}, \mathrm{pH}$ 7.2). Cells were harvested by scraping the dishes with a rubber policeman.

Cultures prepared as described above contain both fibroblasts and myotubes, but several studies (Fluck and Strohman, 1973; Wilson et al., 1973; Koenig and Vigny, 1978; Rotundo and Fambrough, 1979) have reported that fibroblasts have no measurable AChE activity. Thus 
virtually all of the AChE in these cultures is associated with the myotubes.

Sequential extraction of globular, asymmetric, and nonextractable AChE. Extraction was carried out using a modification of the method described by Younkin et al. (1982). Globular forms of AChE were extracted initially (S1) from washed cells using low ionic strength buffer (LIB) - a $10 \mathrm{~mm}$ phosphate buffer, $\mathrm{pH} 7$, containing $1 \%$ Triton X-100, $5 \mathrm{~mm} N$-ethylmaleimide, $2 \mathrm{~mm}$ benzamidine, and $10 \mathrm{~mm}$ EGTA. The pellet remaining after the initial extraction was extracted once more with LIB to remove residual globular forms (S2). Asymmetric forms (S3) then were obtained by extracting once with high ionic strength buffer (HIB), a buffer identical to LIB except that it contained additionally $1.0 \mathrm{M} \mathrm{NaCl}$. The pellet remaining after these three extractions was homogenized in HIB, and the homogenate $(\mathrm{H} 4)$ was assayed to evaluate the AChE that was not extracted. Previous work has shown that this procedure extracts $92 \pm 4 \%$ of the activity present in the initial homogenate (Brockman et al., 1982). $N$-Ethylmaleimide, benzamidine, and EGTA were included in both extraction buffers to inhibit proteolytic degradation of AChE (Younkin et al., 1982). All homogenizations were carried out in $1.25 \mathrm{ml}$ of buffer using a Polytron (Brinkman Instruments) equipped with a Kinematica generator run at a setting of 8 for $10 \mathrm{sec}$. To improve recovery, residual homogenate was washed from the Polytron by operating it for $5 \mathrm{sec}$ at the same speed using a fresh $1.25-\mathrm{ml}$ volume of buffer. The original homogenate and the wash were pooled prior to centrifugation to give $2.5 \mathrm{ml}$ of final homogenate. All three centrifugations were carried out for $30 \mathrm{~min}$ at $39,000 \times$ $g$ in a Sorvall centrifuge equipped with an SS/34 rotor.

In several experiments, we evaluated the percentage of enzyme activity due to true $\mathrm{AChE}$ by assaying fractions in the presence and absence of $0.1 \mathrm{~mm}$ tetraisopropyl pyrophosphoramide (iso-OMPA), a specific inhibitor of butyrylcholinesterase. iso-OMPA was added to the samples immediately before assay and the assays were run for a period of $2 \mathrm{hr}$. At 5 days, $70 \%$ of the globular activity and $40 \%$ of the asymmetric activity in both fibrillating and nonfibrillating myotubes were due to true $\mathrm{AChE}$ (resistant to iso-OMPA). At 8 days, $56 \%$ of the asymmetric activity in nonfibrillating myotubes and $83 \%$ of the asymmetric activity in fibrillating myotubes was due to true AChE. At this time, essentially all of the globular enzyme in both fibrillating and nonfibrillating myotubes was due to true AChE. Thus the large increases in globular and asymmetric enzyme activity that occurred in fibrillating myotubes (Figs. 1 and 3) were overwhelmingly due to increases in true $\mathrm{AChE}$.

Separation of individual forms on sucrose gradients. Individual forms of $\mathrm{AChE}$ were separated on sucrose gradients as described previously by Younkin et al. (1982). AChE recovery was evaluated by comparing the AChE activity applied with the summed activities of the gradient fractions and was $69 \pm 2 \%(n=13)$.

$A C h E$ assay. AChE was assayed as described previously (Brockman et al., 1982) using a modification of the radiometric assay developed by Johnson and Russell (1975).

Quantitation of AChE activity. The sequential extrac- tion data on myotube $\mathrm{AChE}$ were used to calculate the amounts of globular ( $\mathrm{S} 1 \pm \mathrm{S} 2)$, asymmetric (S3), and nonextractable AChE. The activities of individual globular and asymmetric forms were obtained from the gra dient profiles by expressing the activity in each gradient fraction as a percentage of the total activity on the gradient and summing the percentages, from minimum to minimum, in each of the peaks corresponding to particular forms.

Morphological studies. On days 5 to 8 of culture, myotubes were examined under a phase contrast microscope fitted with an eyepiece with a $10 \times 10$ grid. Eight fields were examined on each dish. In each field, 30 of the 100 squares were scored on a scale of 0 to 3 . A 0 was scored if there was no myotube observed in the square, a 1 was scored if one-third or less of the square was filled, a 2 was scored if one-third to two-thirds of the square was filled, and 3 was scored if two-thirds or more of the square was filled. These scores then were converted to a percentage of the total surface area covered by myotubes. On each day, three to four dishes of both fibrillating and nonfibrillating myotubes were examined.

Titration of active sites with $O$-ethyl-S $S^{2}$-diisopropylaminoethyl methyl-phosphonothionate (MTP). Homogenates of fibrillating and nonfibrillating myotubes were prepared by homogenizing 5 and 13 dishes (fibrillating and nonfibrillating) of 8-day myotubes in $1 \mathrm{ml}$ of LIB. The homogenate of the fibrillating myotubes then was diluted so that it had the same level of AChE activity as the homogenate from the nonfibrillating myotubes. An identical amount of MTP (estimated to inactivate half of the active sites) was added to the two homogenates and they were assayed periodically to determine the amount of AChE activity remaining. At the concentration of MTP used $\left(7.5 \times 10^{-10} \mathrm{M}\right.$ to $\left.9.8 \times 10^{-10} \mathrm{M}\right)$, a stoichiometric titration is achieved after approximately $120 \mathrm{~min}$.

AChE metabolism. The medium from 7-day-old myotube cultures (conditioned medium) was removed and saved. Fresh medium containing $10 \mu \mathrm{M}$ paraoxon $\left(37^{\circ} \mathrm{C}\right)$ was added and the cultures were incubated for $30 \mathrm{~min}$ to inactivate (diethylphosphorylate) almost all (86\%) of the $\mathrm{AChE}$ activity in the cultures. The cultures then were rinsed three times with $\mathrm{PBS}\left(37^{\circ} \mathrm{C}\right)$ and the original medium was replaced. At various times cells were washed with PBS and harvested. In some experiments, four fractions were obtained by sequential extraction as described above. In others, only two fractions were examined, globular forms (S1) and the homogenate of the pellet left after extraction of globular forms (H2). Extracts were assayed both before and after reactivation of diethylphosphorylated enzyme with pyridine-2-aldoxime methiodide (2-PAM) to determine the amount of enzyme present in the cultures labeled by paraoxon. Reactivation was accomplished by adding $0.2 \mathrm{~mm} 2$-PAM to the sample and allowing it to sit $45 \mathrm{~min}$ at room temperature before diluting and assaying. Previous work (Brockman et al., 1982) has shown that this procedure completely reactivates all diethylphosphorylated enzyme.

Spontaneous reactivation of diethylphosphorylated enzyme. To evaluate the stability of diethylphosphorylated enzyme, 8-day-old myotubes first were treated with 10.0 
$\mu \mathrm{M}$ paraoxon for $30 \mathrm{~min}$. At the same time, cultures were treated with $1.0 \mathrm{~mm} 2,4$-dinitrophenol and $100 \mu \mathrm{g} / \mathrm{ml}$ of cycloheximide to inhibit cellular metabolism. The cultures then were washed three times with PBS and me dium containing $1.0 \mathrm{mM} \mathrm{2,4-dinitrophenol} \mathrm{and} 100 \mu \mathrm{g} /$ $\mathrm{ml}$ of cycloheximide was added. Cultures were harvested immediately after treatment with paraoxon and $2 \mathrm{hr}$ after treatment. Homogenates were prepared in LIB and AChE activity was determined.

Materials. Rats were from Zivic-Miller Co. (Allison Park, PA), Dulbecco's modified Eagle's medium was from Flow Laboratories (McClean, VA), fetal calf serum was from K.C. Biological, Hanks' balanced salt solution and antibiotics were from M.A. Bioproducts, collagenase (CLS II) was from Worthington Biochemical Corp. (Freehold, NJ) [ ${ }^{3} \mathrm{H}$ ] acetylcholine was from New England Nuclear (Boston, MA), iso-amyl alcohol was from Fisher, 4 a20 cocktail was from Research Products International, (Elk Grove Village, IL), N-ethylmaleimide was from Eastman Kodak Co. (Rochester, NY) and pyridine-2zamidine, iso-OMPA, sucrose (grade 1), and Triton $\mathrm{X}$ 100 were from Sigma Chemical Co. (St. Louis, MO).

\section{Results}

Development of the molecular forms of $A C h E$. The results described below were obtained using myotube cultures derived from 20-day rat embryos. The myotubes in these cultures develop as described in earlier studies (Heinemann et al., 1977; Bloch, 1979; Rieger et al., 1980; Brockman et al., 1982) and begin to fibrillate on the 5th day in culture. To evaluate the effect of electromechanical activity on the AChE in these cultures, we prevented fibrillation by adding $1.0 \mu \mathrm{M}$ TTX to the medium bathing half of a large number of matched cultures on day 4 afler the culture medium was changed. At various times thereafter, fibrillating and nonfibrillating cultures were subjected to sequential extraction (Brockman et al., 1982; Younkin et al., 1982) to obtain fractions containing globular, asymmetric, and nonextractable AChE. Figure 1 shows that the enzyme activity in each fraction increased much more rapidly in fibrillating than in nonfibrillating cultures. In both fibrillating and nonfibrillating cultures, globular forms of AChE increased steadily between days 5 and 9 (Fig. $1 A$ ), but the increase was greater in fibrillating myotubes (12-fold) than in TTX-treated myotubes (3-fold). Low levels of asymmetric and nonextractable $\mathrm{AChE}$ were detectable in nonfibrillating cultures, but the dramatic increases in asymmetric and nonextractable $\mathrm{AChE}$ that occurred in fibrillating cultures (14-fold and 3-fold, respectively, between days 5 and 9) did not occur when spontaneous electromechanical activity was blocked with TTX (Fig. $1, B$ and $C$ ). Overall, between days 5 and 9 , total AChE rose 11-fold in fibrillating and only 2 -fold in nonfibrillating myotubes (Fig. 1D).

To evaluate the effect of spontaneous electromechanical activity on individual globular and asymmetric forms, the globular and asymmetric fractions obtained by sequential extraction were applied to sucrose gradients. The gradient profiles obtained from one set of myotube cultures are shown in Figures 2 (5-day cultures) and 3 (8-day cultures). At 5 days, the activities of the 10

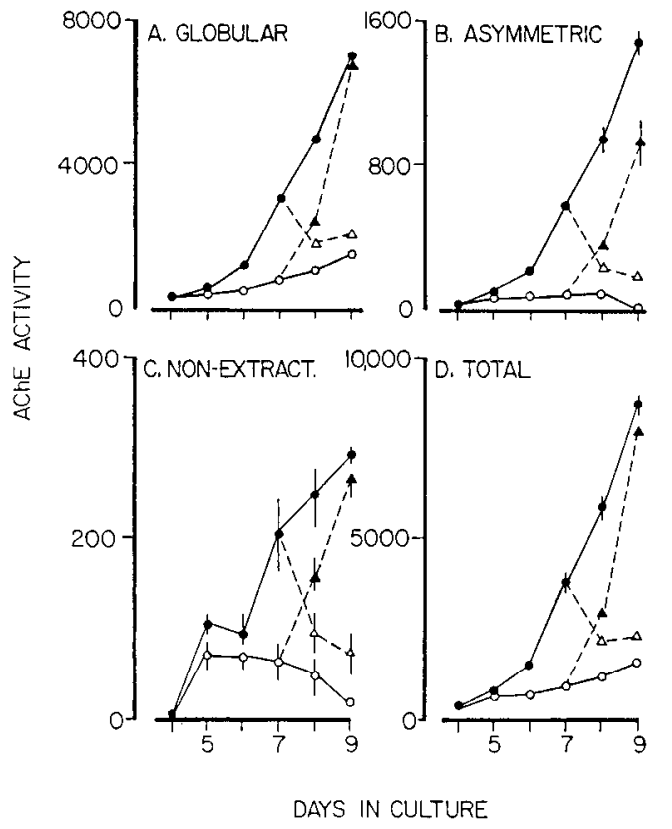

Figure 1. Development of AChE forms in myotube cultures. Myotube cultures at various ages were subjected to sequential extraction. AChE activity then was measured. Globular, asymmetric, nonextractable, and total $\mathrm{AChE}$ are shown in $A, B, C$, and $D$, respectively. Data for untreated myotube cultures (O), myotube cultures treated at day 4 with $1 \mu \mathrm{M}$ TTX (O), TTXtreated myotube cultures washed at day 7 to remove TTX $(\mathbf{A})$, and myotube cultures to which TTX was added at day $7(\triangle)$ are shown. Activity is expressed as picomoles of ACh hydrolyzed $/ \mathrm{min}^{-1} \mathrm{dish}^{-1}$. Each point represents the mean $\pm \mathrm{SEM}$ of three to eight determinations. Where no error is shown the error is less than the size of the symbol.

$\mathrm{S}$ and $4 \mathrm{~S}$ forms were the same in control myotubes (which were just beginning to fibrillate) and in myotubes treated with TTX (Fig. 2, $A$ and $C$ ). Both globular forms increased in fibrillating and nonfibrillating myotubes between 5 and 8 days, but the increases in the fibrillating myotubes were greater. By 8 days, fibrillating myotubes contained 4.8 -fold more $10 \mathrm{~S}\left(\mathrm{G}_{4}\right)$ and 4.3 -fold more $4 \mathrm{~S}$ $\left(\mathrm{G}_{1}\right)$ AchE (Fig. 3, $A$ and $C$ ) than did nonfibrillating myotubes, with total levels of globular forms being 4.4fold greater in fibrillating myotubes. At 5 days, the asymmetric fraction was composed of three forms that sedimented at $16 \mathrm{~S}$ and at approximately $10 \mathrm{~S}$ and $6 \mathrm{~S}$. The activity of each of these forms was essentially identical in fibrillating and nonfibrillating myotubes at this stage (Fig. 2, $B$ and $D$ ). The asymmetric forms in fibrillating myotubes increased dramatically between 5 and 8 days, but there was no appreciable change in any of the asymmetric forms in nonfibrillating myotubes. By 8 days, fibrillating myotubes had 19.3 -fold more $16 \mathrm{~S}\left(\mathrm{~A}_{12}\right)$ AChE, they contained appreciable $12.5 \mathrm{~S}\left(\mathrm{~A}_{8}\right) \mathrm{AChE}$ that was not apparent in the nonfibrillating cells, and they contained increased amounts of the $10 \mathrm{~S}$ form found in the asymmetric fraction (Fig. $3, B$ and $D$ ). At 8 days the fibrillating myotubes had 11.2-fold more total asymmetric $\mathrm{AChE}$ than did nonfibrillating myotubes. The $6 \mathrm{~S}$ form in the asymmetric fraction was difficult to evaluate in fibrillating myotubes, but it clearly did not increase as the other asymmetric forms did. 

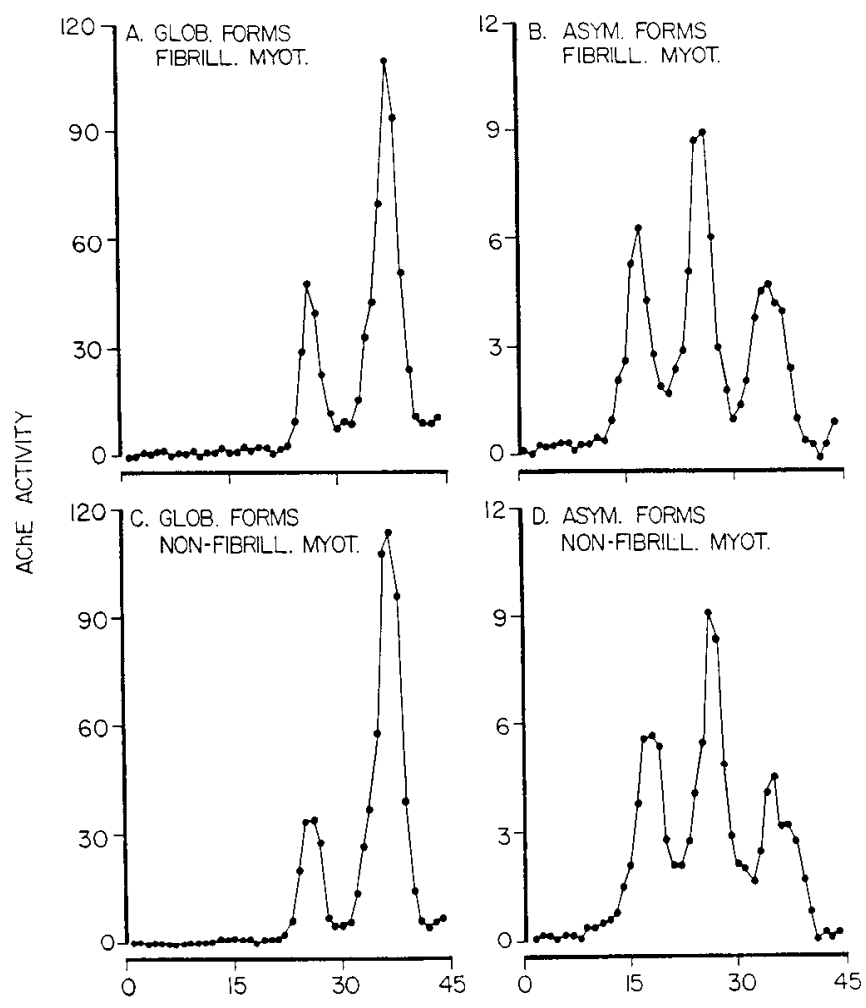

FRACTION NUMBER

Figure 2. Forms of AChE in 5 day myotubes. The gradient profiles shown were obtained by applying fractions obtained by the sequential extraction procedure to sucrose velocity sedimentation gradients. $A$, Globular forms, fibrillating myotubes; $B$, Asymmetric forms, fibrillating myotubes; $C$, Globular forms, nonfibrillating myotubes; $D$, Asymmetric forms, nonfibrillating myotubes. The gradients of globular forms show enzyme with sedimentation coefficients of $10 \mathrm{~S}$ and $4 \mathrm{~S}$ (left to right) and gradients of asymmetric forms show enzyme with sedimentation coefficients of $16 \mathrm{~S}, 10 \mathrm{~S}$, and $6 \mathrm{~S}$. Activity is expressed as picomoles of $\mathrm{ACh}$ hydrolyzed $/ \mathrm{min}^{-1} \mathrm{dish}^{-1}$.

Morphological development. The effect of TTX on AChE was not related to changes in the gross morphological development of the myotubes. The percentage of total surface area of the culture dish covered by myotubes was carefully examined in 5- to 8-day-old cultures as described under "Materials and Methods." The surface area covered by myotubes increased linearly from $13 \%$ to $35 \%$ in both fibrillating and nonfibrillating cultures (Fig. 4).

Reversibility of TTX effect. The effect of TTX was reversible. When cultures that had been exposed to TTX on day 4 were washed on day 7 and bathed in medium obtained from 7-day fibrillating cultures (conditioned medium), globular forms returned to control levels in 2 days (Fig. 1A). Asymmetric (Fig. $1 B$ ) and non-extractable (Fig. $1 C$ ) forms also recovered dramatically over 2 days. We used conditioned medium rather than fresh medium in this experiment because AChE activity decreases when medium that has been conditioned for several days is replaced with fresh medium (Table I). Control experiments established that the increase in AChE activity was due to the resumption of electromechanical activity rather than to factors in the conditioned

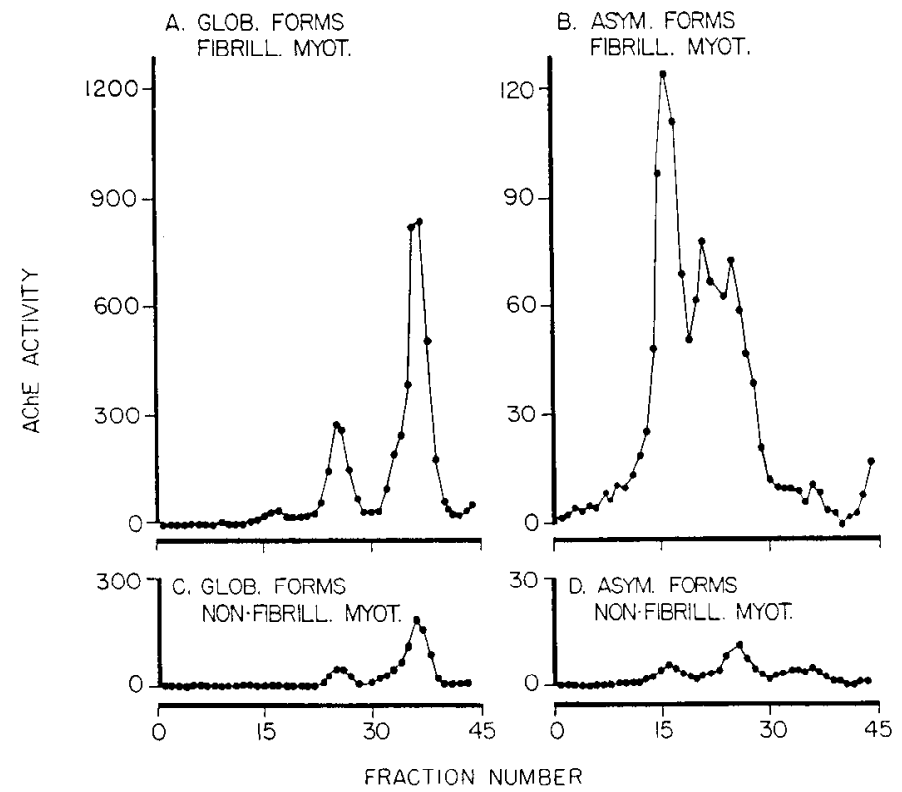

Figure 3. Forms of AChE in 8-day myotubes. The gradient profiles shown were obtained by applying fractions obtained by the sequential extraction procedure to sucrose velocity sedimentation gradients. A, Globular forms, fibrillating myotubes; $B$, Asymmetric forms, fibrillating myotubes; $C$, Globular forms, nonfibrillating myotubes; $D$, Asymmetric forms, nonfibrillating myotubes. The gradients of globular forms show enzyme with sedimentation coefficients of $10 \mathrm{~S}$ and $4 \mathrm{~S}$ (left to right). The gradients of asymmetric forms show enzyme with sedimentation coefficients of $16 \mathrm{~S}, 12.5 \mathrm{~S}$, and $10 \mathrm{~S}$ (left to right) in fibrillating myotubes and $16 \mathrm{~S}, 10 \mathrm{~S}$, and $6 \mathrm{~S}$ in nonfibrillating myotubes. Activity is expressed as picomoles of ACh hydrolyzed $/ \mathrm{min}^{-1} \mathrm{dish}^{-1}$.

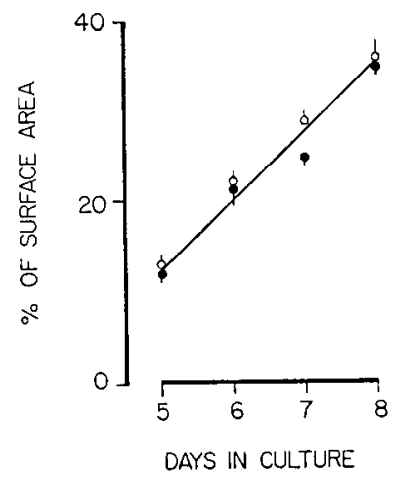

Figure 4. Morphological development of myotubes. The percentage of surface area of the culture dish covered by myotubes was determined by examining cultures with phase contrast microscopy as described under "Materials and Methods." Data from fibrillating myotube cultures $(0)$ and nonfibrillating myotube cultures (O) are shown. Each point represents the mean $\pm \mathrm{SEM}$ of three or four determinations.

medium. When medium from fibrillating myotube cultures was supplemented with TTX and used to replace the medium of the nonfibrillating cultures, AChE activity did not increase (Table I).

Inhibition of fibrillation at 7 days (when cultures were fibrillating vigorously and had developed significant levels of AChE) not only prevented AChE from increasing normally but also caused all fractions to be reduced 
TABLE I

Effect of medium replacement on myotube AChE activity Seven-day myotube cultures were subjected to the treatments indicated. AChE activity was determined on day 8 . The results are expressed as a percentage of the activity present in the untreated (control) cultures and are expressed as the mean \pm SEM.

\begin{tabular}{|c|c|c|c|}
\hline Culture Status & Treatment & $N$ & $\begin{array}{c}\text { Percentage of } \\
\text { Control } \\
\text { AChE }\end{array}$ \\
\hline \multirow[t]{3}{*}{ Fibrillating } & None (control) & 6 & $100.0 \pm 4.9$ \\
\hline & $\begin{array}{l}\text { Washed, original medium } \\
\text { saved and reapplied }\end{array}$ & 3 & $93.9 \pm 0.9$ \\
\hline & $\begin{array}{l}\text { Washed, original medium } \\
\text { discarded and replaced } \\
\text { with fresh medium }\end{array}$ & 6 & $33.9 \pm 2.1$ \\
\hline \multirow[t]{3}{*}{ Nonfibrillating } & None & 5 & $21.7 \pm 1.5$ \\
\hline & $\begin{array}{l}\text { Washed, TTX medium dis- } \\
\text { carded and replaced with } \\
\text { medium from fibrillating } \\
\text { cultures }\end{array}$ & 6 & $49.6 \pm 4.1$ \\
\hline & $\begin{array}{l}\text { Washed, TTX medium dis- } \\
\text { carded and replaced with } \\
\text { medium from fibrillating } \\
\text { cultures that was supple- } \\
\text { mented with TTX }\end{array}$ & 2 & 24.0 \\
\hline
\end{tabular}

within 1 day to levels only slightly higher than those found in cultures where fibrillation had been entirely prevented (Fig. 1). Thus the effect of TTX is reversible, occurs relatively rapidly, and is not critically dependent on the developmental stage of the culture.

Titration of AChE active sites with MTP. The increase in AChE activity associated with fibrillation could be due either to an increase in the activity of individual $\mathrm{AChE}$ molecules or to an increase in the number of molecules present. Vigny et al. (1978) have shown that the stable, irreversible phosphorylating agent MTP can be used to titrate AChE active sites stoichiometrically. MTP binds to AChE active site extremely rapidly (the second-order rate constant is about $1.4 \times 10^{6} \mathrm{~mol}^{-1} \mathrm{sec}^{-1}$ ) and completely irreversibly with each molecule of inhibitor added to the homogenate inactivating one active site. We used MTP to evaluate the number and catalytic activity in AChE active sites in 8-day fibrillating and nonfibrillating myotubes. In these experiments, homogenates of fibrillating and nonfibrillating myotubes having the same AChE activity were prepared as described under "Materials and Methods." An identical amount of MTP (estimated to inactivate half of the active sites) then was added to the two homogenates, and they were assayed periodically for residual $\mathrm{AChE}$ activity. Typical results were shown in Figure 5. It is evident that MTP had an essentially identical effect on the $\mathrm{AChF}$ from fibrillating and nonfibrillating myotubes. This result provides strong evidence that the catalytic activity of individual active sites is essentially identical in fibrillating and nonfibrillating myotubes, so that the increase in $\mathrm{AChE}$ activity associated with fibrillation is due primarily, if not exclusively, to an increase in the number of AChE molecules present. In three experiments on 8-day cultures, each dish of fibrillating myotubes contained $0.53 \pm 0.08 \mathrm{pmol}$ of active sites and each dish of nonfibrillating myotubes contained $0.10 \pm 0.02$ pmol of active sites. The apparent

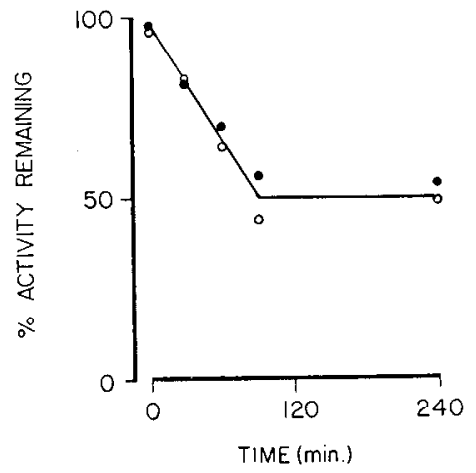

Figure 5. Inactivation of $\mathrm{AChE}$ by MTP. Homogenates containing identical levels of $\mathrm{AChE}$ activity were prepared from fibrillating myotubes (O) and nonfibrillating myotubes (O). An equal amount of MTP was added to each and the remaining amount of active $\mathrm{AChE}$ (expressed as a percentage of initial activity present) was determined at various times.

first-order rate constant for acetylcholine turnover at active sites in fibrillating myotubes $\left(229 \pm 22 \mathrm{sec}^{-1}\right)$ was not different statistically from that in nonfibrillating myotubes $\left(217 \pm 9 \mathrm{sec}^{-1}\right)$. This turnover number for rat myotube AChE corresponds to a value that is one-third to one-half of that reported by Vigny et al. (1978) for rat brain AChE after allowance is made for the different assay conditions used in our study.

Metabolism of $A C h E$. The increased number of AChE molecules in fibrillating myotubes could be due to increased synthesis, decreased turnover, or to some combination of the two. To evaluate AChE metabolism, we examined the enzyme after inactivating it with paraoxon (diethyl $p$-nitrophenyl phosphate). Paraoxon readily penetrates cells and diethylphosphorylates a serine in the active site of AchE, thereby inactivating the enzyme and releasing $p$-nitrophenol. 'The diethylphosphorylated enzyme is stable, but it can be reactivated rapidly and quantitatively with 2-PAM (Brockman et al., 1982; Younkin et al., 1982). Thus one can inactivate $\mathrm{AChE}$ with paraoxon and simultaneously evaluate synthesis (by following the newly synthesized active $\mathrm{AChE}$ ) and turnover (by following the 2-PAM-reactivatable AChE). The combination of diethylphosphorylation and 2-PAM reactivation provides, in effect, an extremely sensitive and highly specific way to label AChE. An attractive feature of this method is that there is no reutilization of label following degradation of labeled AChE or following spontaneous reactivation of inactivated (labeled) AChE because the enzyme is not inactivated (labeled) by diethylphosphate per se.

In homogenates in vitro, the rate of spontaneous reactivation of diethylphosphorylated AChE is about 0.026 $\mathrm{hr}^{-1}$ at $37^{\circ} \mathrm{C}$ (Newman et al., 1983) Therefore, there should be very little generation of active $\mathrm{AChE}$ by reactivation of the labeled pool in short experiments like those described below. To confirm this, control experiments were performed in which cellular metabolism was inhibited as described under "Materials and Methods." The active enzyme that appeared in these cultures after removal of paraoxon represents the maximal amount that could be generated by spontaneous reactivation of diethylphosphorlyated enzyme. Over a 2 -hr period, only 
$104 \mathrm{pmol} \mathrm{min}^{-1} \mathrm{dish}^{-1}$ of active enzyme appeared in the cultures $(n=4)$. This represented only $5.1 \%$ of the total amount of labeled enzyme present immediately after treatment with paraoxon. Thus the spontaneous reactivation of diethylphosphorylated $\mathrm{AChE}$ in cultured myotubes occurs slowly and at essentially the rate observed in vitro.

Control experiments were performed to evaluate the impact of paraoxon on $\mathrm{AChE}$ metabolism. If paraoxon inactivation influenced $\mathrm{AChE}$ metabolism appreciably, then the amount of globular and/or asymmetric AChE present would change. Labeling by paraoxon inactivation is advantageous because the total amount of AChE present can be assessed by assaying $\mathrm{AChE}$ after reactivating the diethylphosphorylated enzyme with 2-PAM. Figure 6 shows that no appreciable change in total globular or asymmetric AChE (measured after 2-PAM reactivation) occurred in either fibrillating or nonfibrillating myotubes over the 2-hr period of our experiments.

Seven-day fibrillating and nonfibrillating cultures were treated with $10 \mu \mathrm{M}$ paraoxon for $30 \mathrm{~min}$ to label (diethylphosphorylate) AChE. This protocol labeled an average of $86 \%$ of the enzyme in the cells. The paraoxon was then washed from the cultures and, at various times, the myotubes were harvested. The cells were homogenized and two fractions were prepared. One contained globular AChE and the other contained enzyme (left after extraction of the globular enzyme) that is almost entirely asymmetric $\mathrm{AChE}$. These fractions were assayed before and after reactivation of the diethylphosphorlyated enzyme with 2-PAM in order to evaluate both newly synthesized (active) AChE and labeled (2-PAMreactivatable) enzyme.

The percentage of labeled globular and asymmetric AChE remaining at various times after labeling is shown in Figure 7. The half-life of globular AChE was shorter in fibrillating cells $(85 \mathrm{~min})$ than in nonfibrillating cells (122 $\mathrm{min}$ ), but the difference was not statistically signif-

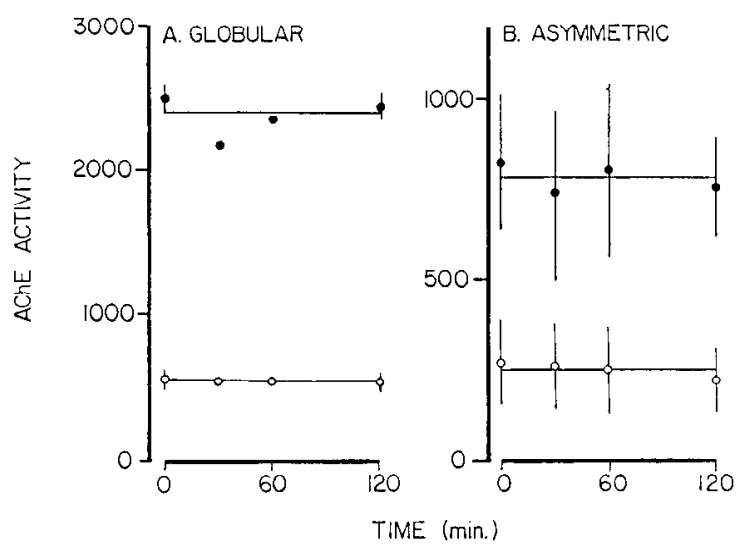

Figure 6. Total levels of AChE present after paraoxon labeling. Total AChE activity (labeled plus unlabeled) present in the myotubes was determined at various times after paraoxon labeling. Data from fibrillating myotube cultures (O) and nonfibrillating myotube cultures (O) are shown. A, Globular forms; $B$, Asymmetric forms. Activity is expressed as picomoles of ACh hydrolyzed $/ \mathrm{min}^{-1} \mathrm{dish}^{-1}$. Each point represents the mean $\pm \mathrm{SEM}$ of three determinations. Where no error is shown the error is less than the size of the symbol.

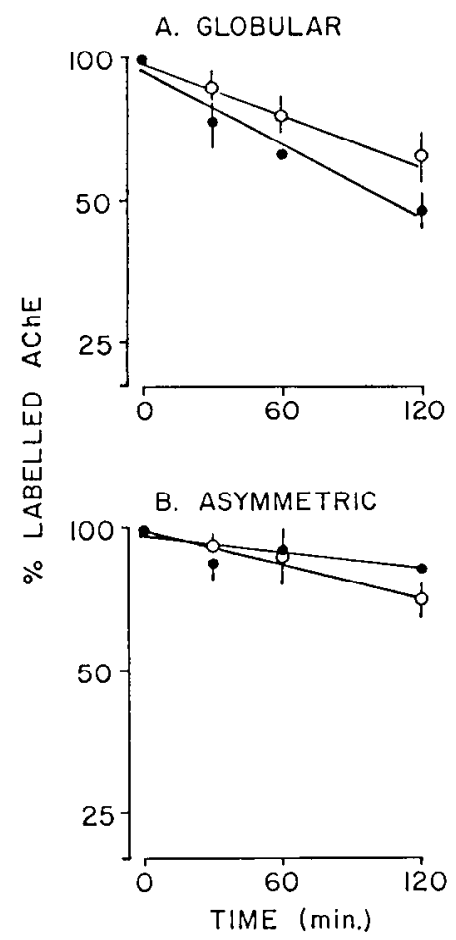

Figure 7. Disappearance of labeled AChE. At various times after paraoxon treatment the amount of labeled AChE present in the myotube cultures was determined. The results are expressed as a percentage of the initial amount of labeled $\mathrm{AChE}$ present. Data for fibrillating myotube cultures $(\mathbf{)})$ and nonfibrillating myotube cultures (O) are shown. A, Globular forms; $B$, Asymmetric forms. Each point represents the mean $\pm \mathrm{SEM}$ of three determinations.

icant. The turnover rate of asymmetric $\mathrm{AChE}$ was too slow to assess accurately in the 2 -hr interval over which our measurements were made. In Figure 7 it appears that asymmetric AChE turns over more slowly in fibrillating than in nonfibrillating myotubes. The difference between fibrillating and nonfibrillating cells was not significant, however, and, in a second series of experiments in which turnover rates were approximately the same as those in Figure 7 (data not shown), asymmetric AChE turned over more rapidly in fibrillating than in nonfibrillating myotubes (again the difference was not significant).

The newly synthesized $\mathrm{AChF}$ measured at various times after inactivation is shown in Figure 8. It is apparent that newly synthesized globular and asymmetric forms both appear more rapidly in fibrillating than in nonfibrillating cells. Based on the reappearance of AChE in the first $60 \mathrm{~min}$, it appears that globular forms are synthesized 7 times faster in fibrillating myotubes than in nonfibrillating myotubes and that asymmetric forms are synthesized 6 times faster in fibrillating than in nonfibrillating myotubes.

The "asymmetric" AChE evaluated above was the enzyme left after a single extraction of globular forms. This enzyme consists mostly of soluble asymmetric forms but also contains nonextractable $\mathrm{AChE}$ and some residual globular forms. To rule out the possibility that increased synthesis was occurring entirely in the contaminating forms, we examined the rate of synthesis of the soluble asymmetric AChE obtained after extracting twice 
to remove globular forms (see "Materials and Methods" for details of the extraction procedure). Figure 9 clearly shows that soluble asymmetric $\mathrm{AChE}$ is synthesized more rapidly in fibrillating than in nonfibrillating myotubes. Following inhibition, asymmetric AChE appeared about 4 times faster in fibrillating than in nonfibrillating myotubes over the first $2 \mathrm{hr}$ of recovery.

\section{Discussion}

On the basis of evidence obtained in earlier work, we proposed (Collins and Younkin, 1982) that the electromechanical activity set up in mammalian skeletal muscle

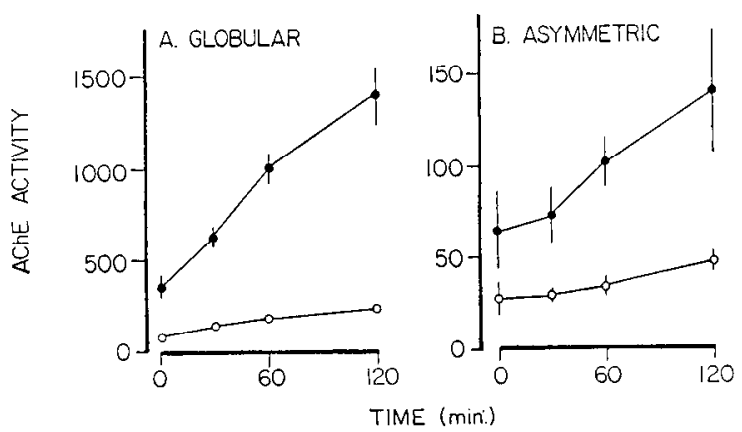

Figure 8. Appearance of labeled AChE. At various times after paraoxon treatment the amount of unlabeled AChE present in the myotube cultures was determined. Data for fibrillating myotube cultures $(O)$ and nonfibrillating myotube cultures $(O)$ are shown. A, Globular forms. $B$, Asymmetric forms. The results are expressed as picomoles of ACh hydrolyzed $/ \mathrm{min}^{-1}$ dish $^{-1}$. Each point represents the mean \pm SEM of three determinations. Where no error is shown the error is less than the size of the symbol.

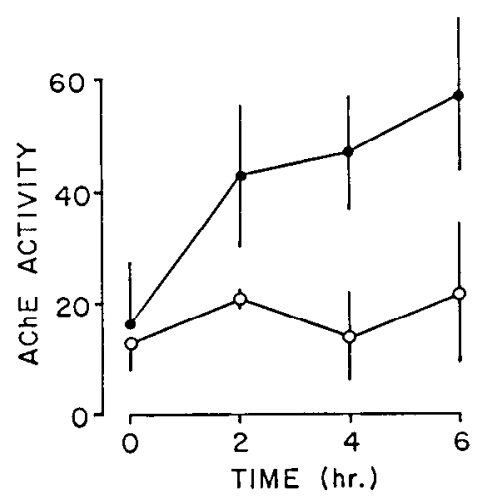

Figure 9. Appearance of unlabeled asymmetric AChE. At various times after paraoxon treatment the amount of unlabeled AChE present in the fraction from the sequentia' extraction procedure that contained only asymmetric forms was determined. Data for fibrillating myotube cultures $(0)$ and nonfibrillating myotube cultures $(O)$ are shown. The results are expressed as picomoles of ACh hydrolyzed $/ \mathrm{min}^{-1} \mathrm{dish}^{-1}$. Each point represents the mean \pm SEM of three determinations. Note that the absolute increase in asymmetric AChE activity here is less than that seen in the first set of experiments (Fig. 8). This occurred, in part, because of the removal of residual globular forms and nonextractable $\mathrm{AChE}$ and, in part, because the cells in the second set of experiments contained less asymmetric AChE ( 776 versus 256 picomoles $/ \mathrm{min}^{-1} \mathrm{dish}^{-1}$ for fibrillating myotubes and 250 versus 110 picomoles $/ \mathrm{min}^{-1} \mathrm{dish}^{-1}$ for nonfibrillating myotubes). by motor neurons increases nonjunctional AChE by increasing the synthesis of the globular forms of the enzyme. We suggested, in addition, that increased synthesis of asymmetric forms might contribute to the effect of motor neurons on junctional AChE. In this study, we have tested the hypothesis that electromechanical activity increases the synthesis of globular and asymmetric AChE by comparing fibrillating rat myotubes with TTXtreated nonfibrillating myotubes.

Our experiments confirm many of the results reported by Rieger et al. (1980) in their study of the effect of fibrillation on cultured embryonic rat myotubes. Like Rieger et al. (1980) we find (1) that a large increase occurs in the $16 \mathrm{~S}\left(\mathrm{~A}_{12}\right)$ form of $\mathrm{AChE}$ (19-fold in our study) between 5 and 8 days when fibrillation occurs in the myotubes, (2) that this increase in $\mathrm{A}_{12} \mathrm{AChE}$ is prevented or reversed by preventing or stopping fibrillation with TTX, and (3) that the effect of TTX is reversible (Figs. 1 to 3 ).

In their study, Rieger et al. (1980) concentrated on the $\mathrm{A}_{12}$ form of $\mathrm{AChE}$ and did not report on the other forms of the enzyme. Our data indicate that the $4 \mathrm{~S}\left(\mathrm{G}_{1}\right), 10 \mathrm{~S}$ $\left(\mathrm{G}_{4}\right)$, and $12.5 \mathrm{~S}\left(\mathrm{~A}_{8}\right)$ forms are also affected when fibrillation is blocked with TTX. Between 5 and 8 days in culture, fibrillating myotubes develop over 4 times more $4 \mathrm{~S}\left(\mathrm{G}_{1}\right)$ and $10 \mathrm{~S}\left(\mathrm{G}_{10}\right)$ AChE than do nonfibrillating myotubes, and the $12.5 \mathrm{~S}\left(\mathrm{~A}_{\diamond}\right)$ form, which is not detectable in nonfibrillating myotubes, develops appreciably in fibrillating cells between 5 and 8 days (Figs. 2 and 3 ). Thus inhibition of fibrillation with TTX substantially impairs the development of both globular $\left(\mathrm{G}_{4}\right.$ and $\left.\mathrm{G}_{1}\right)$ and asymmetric $\left(\mathrm{A}_{12}\right.$ and $\left.\mathrm{A}_{8}\right)$ forms of $\mathrm{AChE}$ with a much larger and more dramatic effect being exerted on the asymmetric forms.

Our results differ from those reported by Rieger et al. (1980) in one relatively minor way. In their study, no asymmetric forms were detected when fibrillation was prevented. In the asymmetric fraction from our nonfibrillating myotubes (Figs. $2 D$ and $3 D$ ), three distinct forms were clearly present: the classical $16 \mathrm{~S}\left(\mathrm{~A}_{12}\right)$ form and two other forms that sedimented at about $10 \mathrm{~S}$ and $6 \mathrm{~S}$. Gradient fractions were not assayed in the presence of iso-OMPA, so that it is possible that the two smaller forms in the asymmetric fraction are not true AChE. It appears that the $10 \mathrm{~S}$ form in the asymmetric fraction is not simply residual globular enzyme because it, like the $16 \mathrm{~S}\left(\mathrm{~A}_{12}\right)$ and $12.5 \mathrm{~S}\left(\mathrm{~A}_{8}\right)$ forms, aggregates and migrates to the bottom of low ionic strength sucrose gradients whereas the $10 \mathrm{~S}$ form in the globular fraction does not (Brockman et al., 1982). We have not yet investigated the $6 \mathrm{~S}$ form in the asymmetric fraction in this manner. In our experimentation, we isolated the asymmetric fraction before separating the individual asymmetric forms by velocity sedimentation. Rieger et al. (1980) separated both globular and asymmetric forms on the same gradient. This makes it impossible to detect the unusual 10 $\mathrm{S}$ and $6 \mathrm{~S}$ forms and difficult to detect the classical $16 \mathrm{~S}$ form. Figure 2, $C$ and $D$, shows that the $16 \mathrm{~S}$ form in nonfibrillating myotubes is a tiny fraction of the $10 \mathrm{~S}$ and $4 \mathrm{~S}$ forms. It seems quite possible that this form was lost in the base line when it was examined on the same gradient as the more abundant globular forms. Our study 
was carried out on myotubes derived from 20-day embryos whereas Rieger et al. (1980) examined myotubes derived from 18-day embroys. This may also account for the minor difference in the two studies.

The AChE level in 7-day fibrillating myotubes decreases when the culture medium is replaced, presumably because of the removal of factor(s) released into the medium by fibrillating cells. This raised the possibility that the effect of electromechanical activity is mediated by factor(s) released into the medium. Table I shows, however, that conditioned medium by itself had little effect on AChE levels in nonfibrillating myotubes. Thus electromechanical activity and conditioned medium are both required for the development of maximal $\mathrm{AChE}$ activity.

Our morphological data (Fig. 4) show that the effect of TTX is not due to impairment of the gross morphological development of the myotubes. The experiments in which we used MTP to titrate AChE active sites (Fig. 5) establish that the increased AChE activity caused by fibrillation is due to an increase in the number of $\mathrm{AChE}$ molecules present and not to an increase in the rate at which individual $\mathrm{AChE}$ molecules turn over acetylcholine.

Our examination of AChE metabolism shows that both globular and asymmetric forms of AChE are synthesized more rapidly in fibrillating than in TTX-treated nonfibrillating myotubes. Newly synthesized globular forms of $\mathrm{AChE}$ accumulated much more rapidly in fibrillating than in nonfibrillating myotubes (Fig. 8), and the turnover of these forms was, if anything, more rapid in fibrillating than in nonfibrillating cells (Fig. 7); thus the increased level of globular $\mathrm{AChE}$ in fibrillating myotubes appears to be due entirely to increased synthesis. The asymmetric forms turned over very little in the 2 -hr interval over which metabolism was measured; therefore the turnover of this pool could not be accurately measured. The increased accumulation of newly synthesized asymmetric forms that was observed in fibrillating myotubes (Figs. 8 and 9) clearly indicates that increased synthesis contributes to the increased level of asymmetric forms in fibrillating myotubes.

There have been two studies showing that electromechanical activity decreases the synthesis of acetylcholine receptors in mammalian skeletal muscle (Reiness and Hall, 1977; Linden and Fambrough, 1979). Our data provide strong evidence that electromechanical activity increases the synthesis of globular and asymmetric forms of $\mathrm{AChE}$. It appears, therefore, that electromechanical activity can regulate the synthesis of the functionally important surface proteins in skeletal muscle in either a positive or a negative direction.

\section{References}

Bloch, R. J. (1979) Dispersal and reformation of acetylcholine receptor clusters of cultured rat myotubes treated with inhibitors of energy metabolism. J. Cell Biol. 82: 626-643.

Bon, S., M. Vigny, and J. Massoulié (1979) Asymmetric and globular forms of acetylcholinesterase in mammals and birds. Proc. Natl. Acad. Sci. U. S. A. 76: 2546-2550.

Brockman, S. K., R. J. Przybylski, and S. G. Younkin (1982)
Cellular localization of the molecular forms of acetylcholinesterase in cultured embryonic rat myotubes. J. Neurosci. 2: 1775-1785.

Carter, J. L., and S. Brimijoin (1981) Effects of acute and chronic denervation on release of acetylcholinesterase and its molecular forms in rat diaphragms. J. Neurochem. 36: 1018-1025.

Collins, P. L., and S. G. Younkin (1982) Effect of denervation on the molecular forms of acetylcholinesterase in rat diaphragm. J. Biol. Chem. 257: 13638-13644.

Drachman, D. B. (1972) Neurotrophic regulation of muscle cholinesterase: Effects of botulinum toxin and denervation. J. Physiol. (Lond.) 226: 619-627.

Fluck, R. A., and R. C. Strohman (1973) Acetylcholinesterase activity in developing skeletal muscle cells in vitro. Dev. Biol. 33: $417-428$.

Heinemann, S. S., R. Bevan, J. Kullberg, J. Lindștrom, and J. Rice (1977) Modulation of acetylcholine receptor by antibody against the receptor. Proc. Natl. Acad. Sci. U. S. A. 74: 30903094 .

Inestrosa, N. C., B. U. Ramirez, and H. L. Fernandez (1977) Fiffect of denervation and of axoplasmic transport blockage on the in vitro release of muscle endplate AChE. J. Neurochem. 28: 941-945.

Inestrosa, N. C., C. G. Reiness, L. F. Reichardt, and Z. W. Hall (1981) Cellular localization of the molecular forms of acetylcholinesterase in rat pheochromocytoma PC12 cells treated with nerve growth factor. J. Neurosci. 1: 1260-1267.

Johnson, C. D., and R. L. Russell (1975) A rapid, simple radiometric assay for cholinesterase, suitable for multiple determinations. Anal. Biochem. 64: 229-238.

Koenig, J., and M. Vigny (1978) Neural induction of the $16 \mathrm{~S}$ acetylcholinesterase in muscle cell cultures. Nature 271: $75-$ 77.

Lazar, M., and M. Vigny (1980) Modulation of the distribution of acetylcholinesterase molecular forms in a murine neuroblastoma $\times$ sympathetic ganglion cell hybrid cell line. J. Neurochem. 35: 1067-1079.

Linden, D. C., and D. M. Fambrough (1979) Biosynthesis and degradation of acetylcholine receptors in rat skeletal muscles, effect of electrical stimulation. Neuroscience 4: 527-538.

Lomo, T., and J. Rosenthal (1972) Control of ACh sensitivity by muscle activity in the rat. J. Physiol. (Lond.) 221: 493513.

Lomo, T., and C. R. Slater (1980) Control of junctional acctylcholinesterase by neural and muscular influences in the rat. J. Physiol. (Lond.) 303: 191-202.

McCarty, K. S., Jr., R. T. Vollmer, and K. S. McCarty (1974) Improved computer program data for the resolution and fractionation of macromolecules of isokinetic sucrose density gradient sedimentation. Anal. Biochem. 61: 165-183.

Newman, J. R., J. B. Virgin, L. H. Younkin, and S. G. Younkin (1983) Turnover of acetylcholinesterase in innervated and denervated rat diaphragm. J. Physiol. (Lond.), in press.

Reiness, C. G., and Z. W. Hall (1977) Electrical stimulation of denervated muscles reduces incorporation of methionine into ACh receptor. Nature 268: 655-657.

Rieger, F., J. Koenig, and M. Vigny (1980) Spontaneous contractile activity and the presence of the $16 \mathrm{~S}$ form of acetylcholinesterase in rat muscle cells in culture: Reversible suppressive action of tetrodotoxin. Dev. Biol. 76: 358-365.

Rotundo, R. L., and D. M. Fambrough (1979) Molecular forms of chicken embryo acetylcholinesterase in vitro and in vivo. J. Biol. Chem. 254: 4790-4799.

Rotundo, R. I.., and D. M. Fambrough (1980) Synthesis, transport and fate of acetylcholinesterase in cultured chick embryo muscle cells. Cell 22: 583-594.

Rubin, L. L., S. M. Schuetze, C. L. Weill, and G. D. Fischbach 
(1980) Regulation of acetylcholinesterase appearance at neuromuscular junctions in vitro. Nature 283: 264-267.

Taylor, P. B., F. Rieger, M. L. Shelanski, and L. A. Greene (1981) Cellular localization of the multiple molecular forms of acetylcholinesterase in cultured neuronal cells. J. Biol. Chem. 256: 3827-3830.

Vigny, M., S. Bon, J. Massoulié, and F. Leterrier (1978) Active site catalytic efficiency of acetylcholinesterase molecular forms in Electrophorus, Torpedo, rat, and chicken. Eur. J.
Biochem. 85: 317-323.

Wilson, B. W., P. S. Nieberg, C. R. Walker, T. A. Linkhart, and D. M. Fry (1973) Production and release of acetylcholinesterase by cultured chick embryo muscle. Dev. Biol. 33: 285299.

Younkin, S. G., C. Rosenstein, P. L. Collins, and T. L. Rosenbcrry (1982) Cellular localization of the molecular forms of acetylcholinesterase in rat diaphragm. J. Biol. Chem. 257: $13630-13637$. 